\title{
Introduction to the focused section on new trends in modelling and simulation for intelligent robotics
}

\author{
Jin Wang ${ }^{1} \cdot$ Silu Chen ${ }^{2} \cdot \mathrm{Kun} \mathrm{Bai}^{3} \cdot{\text { Bin } \mathrm{He}^{4} \cdot \mathrm{Howard}^{5}{ }^{5} \cdot \text { Jinchuan Zheng }}^{6}$ \\ Published online: 21 October 2021 \\ (c) The Author(s), under exclusive licence to Springer Nature Singapore Pte Ltd. 2021
}

\begin{abstract}
Advanced functional materials, drives and transmissions, sensors and mechanisms provide solid foundations for developing intelligent robots, for which modelling and simulation tools have contributed greatly to these developments, and facilitated deployments and applications of intelligent robots. Motivated by rapid developments of sensor fusion, intelligent control and machine learning tools and their widely availability at low cost, this special issue aims at disseminating new modelling and simulation methods for developing intelligent robotic systems and their applications. With the emerging applications to robotics, this focused section competitively selects the 10 research papers covering a spectrum of modeling of novel single robots, human-robot interactive systems, and even robotic automation process, which includes modeling of biped and wearable robots, parallel mechanisms, autonomous robots, and robot-environment interaction, as well as modeling with robot visions.
\end{abstract}

\section{Modeling of biped and wearable robots}

Biped robot is a bilaterally symmetric walking mechanism, which can be divided into two parts by a sagittal plane such that each divided part is mirror image of other original part. The paper entitled "Using bilateral symmetry of the

Jin Wang
dwjcom@zju.edu.cn
Silu Chen
chensilu@ nimte.ac.cn
Kun Bai
kbai@ hust.edu.cn
Bin He
mehebin@ shu.edu.cn
Howard Li
howard@ unb.ca
Jinchuan Zheng
jzheng@swin.edu.au
Zhejiang University, Hangzhou, China
2 Ningbo Institute of Materials Technology and Engineering,
Chinese Academy of Sciences, Ningbo, China
3 Huazhong University of Science and Technology, Wuhan,
China
$4 \quad$ Shanghai University, Shanghai, China
5 University of New Brunswick, Fredericton, Canada
$6 \quad$ Swinburne University of Technology, Melbourne, Australia

biped robot mechanism for efficient and faster optimal gait learning on uneven terrain" describes the relation between dynamically similar right gait (RG) and left gait (LG) of a 14 degrees of freedom (DOF) biped robot on uneven terrain by using the dynamic similarity principle of gaits (DSPG). The proposed DSPG is validated by generating the optimal $\mathrm{RG}$ of a 14-DOF biped robot using a genetic algorithm (GA) and utilizing DSPG to obtain corresponding dynamically similar LG. Simulation experiments of the bipedal walk on uneven terrain is validated by generating a primitive walk dataset for a 14-DOF biped robot and learning it by a feed forward neural-network (NN). Using the trained NN and DSPG, biped robot can successfully walk on 3D uneven ground by generating real-time optimal gaits.

Wearable exoskeletons have attracted much attention for decades, which naturally fit human bodies and provide strength supplementary. The paper entitled "Model-free fractional-order adaptive back-stepping prescribed performance control for wearable exoskeletons" presents a novel modelfree fractional-order adaptive back-stepping control scheme for the wearable exoskeletons with constraints on the joint angle tracking errors. Firstly, the ultra-local model based intelligent proportion integration differentiation (iPID) control is combined with time delay estimation (TDE) to realize a model-free feature, and the precise modeling process can be abandoned. Then, an adaptive back-stepping controller is introduced to handle with the estimation errors of TDE. Control behavior is greatly improved by introducing 
the fractional-order calculus and prescribed performance is guaranteed by the log-type barrier Lyapunov function implemented in the back-stepping approach. In addition, system stability is verified via Lyapunov stability theory. Finally, to demonstrate the effectiveness of the proposed control scheme, a 5-DOF wearable exoskeleton virtual prototyping is designed and transferred as the controlled plant for visualization simulation.

\section{Modeling of parallel mechanisms}

The diversity of special applications requires designs of novel mechanisms beyond the commonly seen articulated robots and delta robots. The modeling of various parallel robots becomes a hot topics in recent years. The paper entitled "Design of 4PUS-PPPS redundant parallel mechanism oriented to the visual system of flight simulator" presents a novel parallel mechanism which can realize large movement angle around the horizontal axis of the aircraft, compared with conventional Stewart platform. The flight attitude data of the aircraft are acquired and processed by filtering algorithms in the software. And three steering gears that simulate the action of the moving platform are controlled by these processed data. In this way, the simulation control of system is achieved with integration of both virtual and actual information.

\section{Modeling of robot-environment interacting systems}

Till now, the applications of robots have been extended from wielding, pick-and-place applications to the applications such as surface finishing and haptics. It becomes much more challenging to model such process in the sense that the robot are closely interacting with the external during operation. The paper entitled "Inverse input prediction model for robotic belt grinding" presents an inverse input prediction model, which calculate the optimal input parameters for robotic abrasive belt grinding in two steps. First, the forward process is to establish the correspondence between known processing parameters and the unknown grinding depth by Support Vectors Regression. Second, the inverse process of dynamic grid search (DGS) method is proposed to predict the optimal processing parameters according to the required grinding depth. The proposed method can be used to find the stable and reliable sequence of continuous path grinding parameters by given the desired grinding depth.

Haptic interfaces enable manual interaction of a human operator with virtual or remote environments, which are important devices in many applications such as teleoperated remote systems, virtual reality, etc. The paper entitled "Time-delayed dual-rate haptic rendering: stability analysis and reduced order modeling" presents the analysis of the impact of time delay on the stability of dual-rate haptics controller using an exact discrete-time method and an equivalent continuous-time method. Numerical simulations are further employed to assess the effect of the combined presence of time delay and dual-rate sampling on the stability bounds of such controllers. The analysis reveals that the stability bounds of the dual-rate haptics controller depend strongly on the value of time delay relative to the sample rates of the controller. This work provides a design guideline for practitioners of haptic interfaces.

\section{Modeling and path planning of mobile robots}

Autonomous robots can be used for elder care, surveillance, mine hunting, automatic inspection, ocean exploration and many other challenging tasks. There is a growing demand and interest in the modeling, path planning, and navigation techniques of autonomous robots.

The article "Self-E-a self-driving wheelchair for elders and physically challenged" describes a self-driving wheelchair that can be used to assist seniors and people with disabilities. The wheelchair can navigate to the goal location without the user's guidance. The modelling, design, simulation, implementation, and testing are presented. The performance of the wheelchair is validated through experiments. The article "Trajectory planning for mobile manipulators including manipulability percentage index" addresses path planning for mobile manipulators. An operational target point is the goal to be reached by the end-effector of a mobile manipulator. The article discusses feasible trajectories to approach the target point. A singularity avoidance metric named Manipulability Percentage Index (MPI) is introduced to guarantee reachability. It also illustrates how to strike a balance between the minimum time required to complete the task and the MPI index value. The article "A novel solution with rapid Voronoibased coverage path planning in irregular environment for robotic mowing systems" presents a novel coverage planning method for robotic mowers. The path planning algorithm is based on the Boustrophedon motion and the rapid Voronoi diagram. The Voronoi paths are designed to reduce the computational cost. The performance of the proposed algorithm is compared to conventional coverage planning algorithms in terms of time efficiency, coverage, repetition, and robustness. Field tests are conducted 
to demonstrate the performance of the proposed planning algorithm.

\section{Modeling and applications with robot vision}

Vision sensor can perceive the environment in a non-contact way and obtain real-time information with robust anti-interference performance. The theories and applications of robot vision have also become the research highlights and directions to further improve the intelligence of robots in recent years. The paper entitled "Feature-set characterization for target detection based on artificial color contrast and principal component analysis with robotic tealeaf harvesting applications" presents an improved artificial color contrast model with principal component analysis for color feature classification and target detection of intelligent tendertealeaf harvesting robots which require precisely detecting randomly distributed targets in closely similar background. An adaptive width optimization algorithm and 3D rectangular/ellipsoidal boundary shapes for feature-set characterization are also developed to recognize visual target accurately using limited training dataset. As verified experimentally with 120 images under different daylight and weather conditions, the proposed visual target detection method is capable to confront the changing outdoor environments effectively.

The paper "Vision-guided fine-operation of robot and its application in eight-puzzle game" provides a robotic handeye coordination method with the application in playing an eight-puzzle game. A lightweight visual image segmentation and recognition algorithm is developed to detect the position, angle, and layout of the eight-puzzle board. Considering the optimal operation sequence of eight-puzzle game searched by A* algorithm, a visual feedback motion planning method based on the improved kinematics model is established for the robot to move the tiles correctly. The superb experimental performance of eight-puzzle board positioning, digital recognition and tile manipulation demonstrates that the robot can perform autonomous decisions and accurate operations based on vision.

\section{Summary and acknowledgement}

This focused section disseminates the most recent advances on modeling of robot manipulators and systems, humanrobot interaction, and robot automation processes with visions or even sensor fusion techniques. We hope this issue will provides an inspiration for the researchers to continue push forward the research on this field, probably by utilizing the rapid development tools on big data, cloud computing and deep learning. Finally, we would like to acknowledge the great efforts and contributions by the authors and anonymous reviewers towards this focused section. We would also like to extend our sincere appreciations to the editor-in-chief and officers of IJIRA that made this focused section possible.

Publisher's Note Springer Nature remains neutral with regard to jurisdictional claims in published maps and institutional affiliations.

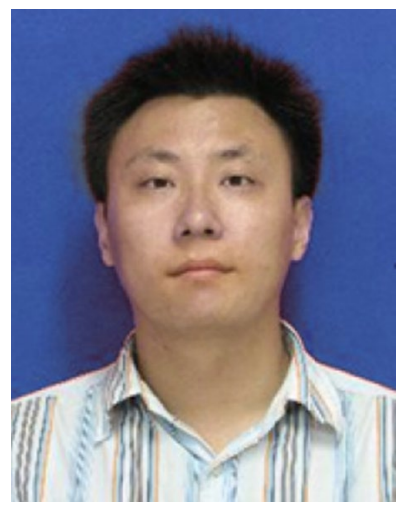

Jin Wang received the B.Eng. degree in mechatronic engineering and the Ph.D. degree in mechanical design and theory from Zhejiang University, Zhejiang, China, in 2003 and 2008, respectively. He was a visiting scholar with the Hong Kong University of Science and Technology, Hong Kong, from 2016 to 2017. He is currently an Associate Professor with the School of Mechanical Engineering, and is the assistant dean of the Institute of robotics, Zhejiang University, Hangzhou, China. His research interests include computer vision, computer graphics, cooperative manipulators control, and robotics.

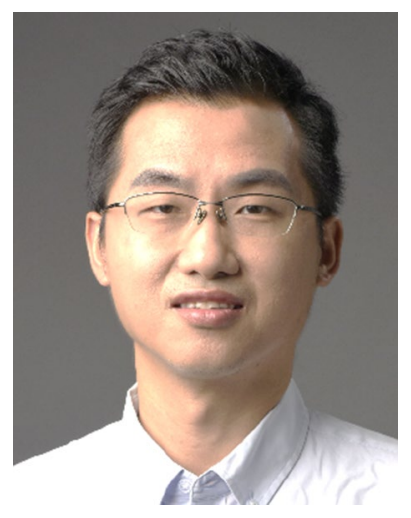

Silu Chen received the B.Eng. and Ph.D. degrees in electrical engineering from the National University of Singapore (NUS), in 2005 and 2010, respectively. From 2010 to 2011, he was with Manufacturing Integration Technology Ltd., a Singapore-based semiconductor machine designer, as a Senior Engineer on motion control. From 2011 to 2017, he was a Scientist with the Mechatronics Group, Singapore Institute of Manufacturing Technology (SIMTech), Agency for Science Technology and Research (A*STAR). Since 2017, he has been with the Ningbo Institute of Materials Technology and Engineering, Chinese Academy of Sciences (CAS), as a Professor. His current research interests include highspeed, high-precision motion control, and industrial automation.

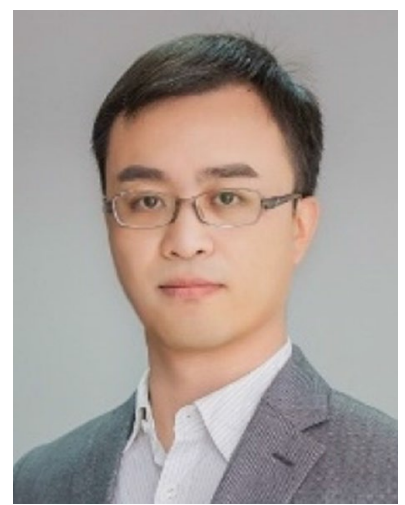

Kun Bai received his B.S. degree in control science and engineering from Zhejiang University, Hangzhou, China, in 2006, and the M.S. and Ph.D. degrees in mechanical engineering from the Woodruff School of Mechanical Engineering, Georgia Institute of Technology, Atlanta, GA, USA, in 2009 and 2012, respectively. $\mathrm{He}$ is currently a Professor with State Key Laboratory of Digital Manufacturing Equipment and Technology, Huazhong 
University of Science and Technology, Wuhan, China. His research interests include smart electromagnetic actuators/sensors and novel applications in robotics and manufacturing. He received ASME DSCD Mechatronics TC Best Paper Award in 2019. He is Guest Editor of IEEE/ASME Trans. on Mechatronics and also Associate Editor for IEEE/ASME International Conference on Advanced Intelligent Mechatronics.

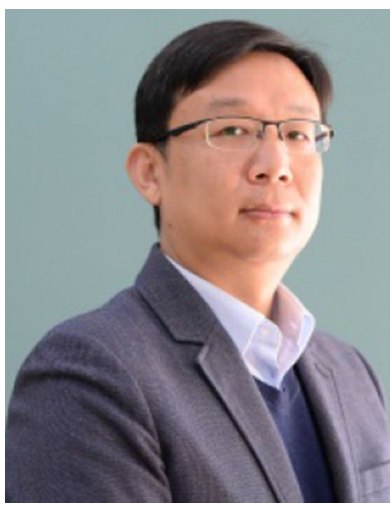

Bin He received his degrees (from Bachelor to Ph.D Degree) from Zhejiang University. Dr.He is a Full Professor and Vice Dean of School of Mechatronic Engineering and Automation, Shanghai University. Dr.He is also the Deputy Director of Shanghai Key Laboratory of Intelligent Manufacturing and Robotics, Director of Robotics and Intelligent Design Institute, Shanghai University. Dr.He is Associate Editor of ASME Journal of Computing and Information Science in Engineering. Dr.He has interests in low-carbon design and manufacturing for carbon neutral, and robotics.

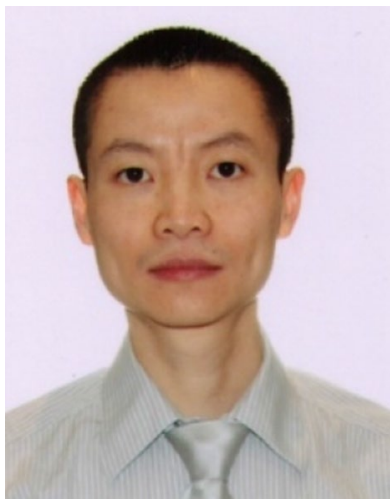

Howard Li received the Ph.D. degree from the University of Waterloo, Canada. He is currently a professor in the Department of Electrical and Computer Engineering, University of New Brunswick, Canada. In addition, worked for Ecole Polytechnique Federale de Lausanne, University of Pavia, University of Waterloo, and University of Guelph. He is a registered professional engineer in the Province of Ontario. He is a member of the IEEE SA Standards Board. He chairs the IEEE Autonomous Robotics Ontology Working Group. He is on the editorial boards of numerous international journals. His research interests include robotics, autonomous systems, unmanned aerial vehicles, unmanned ground vehicles, autonomous underwater vehicles, SLAM, motion planning, control systems, multi-agent systems and artificial intelligence.

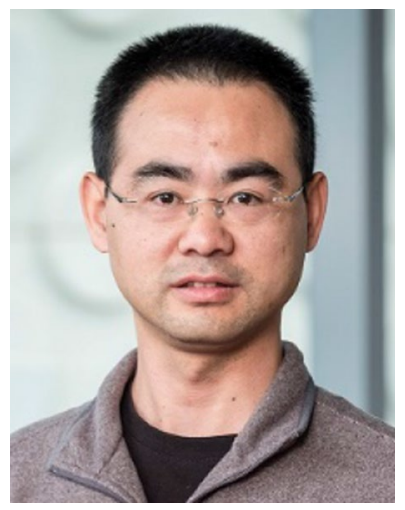

Jinchuan Zheng received the B.Eng. and M.Eng. degrees in mechatronics engineering from Shanghai Jiao Tong University, Shanghai, China, in 1999 and 2002, respectively, and the Ph.D. degree in electrical and electronic engineering from Nanyang Technological University, Singapore, in 2006. In 2005, he joined the Australian Research Council (ARC) Centre of Excellence for Complex Dynamic Systems and Control, School of Electrical and Computer Engineering, the University of Newcastle, Callaghan, NSW, Australia, as a Research Academic. From 2011 to 2012, he was a Staff Engineer with the Western Digital Hard Disk Drive R\&D Center, Singapore. Since 2012, he has been with Swinburne University of Technology, Melbourne, VIC, Australia. He is currently an Associate Professor with the School of Science, Computing and Engineering Technologies. His research interests include high precision motion control systems, electric vehicle control technology, mobile robots, and biomechatronic devices. 